TRANSACTIONS OF THE

AMERICAN MATHEMATICAL SOCIETY

Volume 358, Number 5 , Pages 2057-2069

S 0002-9947(05)03973-5

Article electronically published on December 20, 2005

\title{
ALMOST COMPLEX MANIFOLDS AND CARTAN'S UNIQUENESS THEOREM
}

\author{
KANG-HYURK LEE
}

\begin{abstract}
We present a generalization of Cartan's uniqueness theorem to the almost complex manifolds.
\end{abstract}

\section{INTRODUCTION}

The primary goal of this article is to present a generalization to the almost complex manifolds of the following celebrated theorem of $\mathrm{H}$. Cartan, which is usually called Cartan's uniqueness theorem (see p. 66, [13]).

Theorem 1.1 (H. Cartan). Let $\Omega$ be a bounded domain in $\mathbb{C}^{n}$. If a holomorphic mapping $f: \Omega \rightarrow \Omega$ satisfies that $f(p)=p$ and $d f_{p}=\operatorname{Id}$ for some $p \in \Omega$, then $f$ is the identity mapping.

In order to state the main theorem of this article, we shall introduce the necessary terminology and concepts.

A pair $(M, J)$ is called an almost complex manifold if $M$ is a $C^{\infty}$-smooth real manifold and $J$ is a field of endomorphisms of the tangent bundle $T M$ with $J^{2}=$ -Id, i.e. for each $p \in M, J_{p}: T_{p} M \rightarrow T_{p} M$ is an endomorphism with $J_{p}^{2}=-\mathrm{Id}$. We call $J$ an almost complex structure on $M$. Throughout this paper, by a smooth almost complex manifold we mean a manifold with a $C^{\infty}$-smooth almost complex structure.

Given two almost complex manifolds $(M, J)$ and $\left(M^{\prime}, J^{\prime}\right)$, a $C^{1}$ mapping $f$ from $M$ to $M^{\prime}$ is said to be $\left(J, J^{\prime}\right)$-holomorphic (or simply pseudo-holomorphic, so there is no danger of confusion) if its differential $d f: T M \rightarrow T M^{\prime}$ satisfies

$$
d f \circ J=J^{\prime} \circ d f
$$

on $T M$. If $(M, J)$ is a Riemann surface, $f$ is called a pseudo-holomorphic curve. In the case $(M, J)$ is the unit disc $\mathbf{D}$ in $\mathbb{C}$ with the standard complex structure $J_{s t}$, we call $f$ a pseudo-holomorphic disc. We denote by $\mathcal{O}_{\left(J, J^{\prime}\right)}\left(M, M^{\prime}\right)$ the space of $\left(J, J^{\prime}\right)$-holomorphic mappings from $M$ to $M^{\prime}$.

By the existence theorem of pseudo-holomorphic discs (Nijenhuis and Woolf [15]), we can define the Kobayashi pseudo-distance ([8]) and the Kobayashi-Royden pseudo-metric (16]) for the almost complex manifolds.

Let $(M, J)$ be an almost complex manifold. Given two points $p$ and $q$ in $M$, a finite sequence of pseudo-holomorphic discs $c=\left\{\phi_{j}\right\}_{j=1, \ldots, k} \subset \mathcal{O}_{\left(J_{s t}, J\right)}(\mathbf{D}, M)$

Received by the editors March 12, 2004.

2000 Mathematics Subject Classification. Primary 32H02, 32Q60.

This research was upported in part by Grant KRF-2002-070-C00005 (PI: K.T. Kim) from the Korea Research Foundation.

(C)2005 American Mathematical Society Reverts to public domain 28 years from publication 
is called a chain of pseudo-holomorphic discs from $p$ to $q$ if there are points $p=$ $p_{0}, p_{1}, \ldots, p_{k}=q$ in $M$ and $a_{1}, a_{2}, \ldots, a_{k}$ in $\mathbf{D}$ such that

$$
\phi_{j}(0)=p_{j-1} \quad \text { and } \quad \phi_{j}\left(a_{j}\right)=p_{j}
$$

for $j=1, \ldots, k$. For this chain, we define its length $\ell(c)$ by

$$
\ell(c)=\log \frac{1+\left|a_{1}\right|}{1-\left|a_{1}\right|}+\ldots+\log \frac{1+\left|a_{k}\right|}{1-\left|a_{k}\right|} .
$$

Note that $\log \frac{1+|z|}{1-|z|}$ is the Poincaré distance from 0 to $z$ in D. The Kobayashi pseudo-distance $d_{(M, J)}$ on $(M, J)$ is then defined by

$$
d_{(M, J)}(p, q)=\inf \ell(c),
$$

where the infimum is taken over all chains of pseudo-holomorphic discs from $p$ to $q$. The Kobayashi-Royden pseudo-metric $F_{(M, J)}$ is the infinitesimal version of the Kobayashi pseudo-distance defined by

$$
F_{(M, J)}(p, v)=\inf \left\{\frac{1}{|a|}: \phi \in \mathcal{O}_{\left(J_{s t}, J\right)}(\mathbf{D}, M) \text { with } \phi(0)=p, d \phi(\mathbf{e})=a v\right\},
$$

where $\mathbf{e}$ is the unit vector in $T_{0} \mathbf{D}$ and $p \in M$ and $v \in T_{p} M$. We exploit from [10] and 11 the following properties that are exactly the same as in the integrable case (8] and [16]):

(a) $F_{(M, J)}$ is upper semi-continuous and

$$
d_{(M, J)}(p, q)=\inf \int_{0}^{1} F_{(M, J)}\left(\gamma(t), \gamma^{\prime}(t)\right) d t
$$

where the infimum is taken over all piecewise smooth paths $\gamma:[0,1] \rightarrow M$ with $\gamma(0)=p$ and $\gamma(1)=q$.

(b) Let $f:(M, J) \rightarrow\left(M^{\prime}, J^{\prime}\right)$ be a pseudo-holomorphic mapping. For any points $p$ and $q$ in $M$ and tangent vector $v \in T_{p} M$, we have

$$
d_{\left(M^{\prime}, J^{\prime}\right)}(f(p), f(q)) \leq d_{(M, J)}(p, q)
$$

and

$$
F_{\left(M^{\prime}, J^{\prime}\right)}\left(f(p), d f_{p}(v)\right) \leq F_{(M, J)}(p, v) .
$$

(c) The Kobayashi pseudo-distance $d_{(M, J)}$ is finite and continuous on $M \times M$.

(d) If $d_{(M, J)}$ is a distance, it induces the standard topology on $M$.

We say that $(M, J)$ is (Kobayashi) hyperbolic if $d_{(M, J)}$ is a proper distance. Note that for any neighborhood $U$ of $p \in M$, there is a constant $r>0$ such that the Kobayashi ball $\mathbf{B}_{(M, J)}(p, r)=\left\{q \in M: d_{(M, J)}(p, q)<r\right\}$ is contained in $U$ when $(M, J)$ is hyperbolic.

Now we state our main theorem.

Theorem 1.2. Let $(M, J)$ be a $C^{\infty}$-smooth almost complex manifold. Moreover, $M$ is connected and Kobayashi hyperbolic. Suppose that there is a pseudo-holomorphic mapping $f: M \rightarrow M$ with $f(p)=p$ and $d f_{p}=\mathrm{Id}$. Then $f$ is the identity mapping.

The proof of this theorem appears in Section 5. Sections 2, 3 and 4 contain a regularity theorem and derivative estimates for pseudo-holomorphic mappings which will be used in the proof of Theorem 1.2 


\section{REgularity of PSEUdo-hOLOMORPhiC MAPPINGS}

We now study the smoothness of pseudo-holomorphic mappings. Since the problem is local, we assume that our manifold is a domain in a Euclidean space. Let $(\Omega, J) \subset \mathbb{R}^{2 n}$ and $\left(\Omega^{\prime}, J^{\prime}\right) \subset \mathbb{R}^{2 m}$ be domains with almost complex structures $J \in C^{\infty}(\bar{\Omega})$ and $J^{\prime} \in C^{\infty}\left(\overline{\Omega^{\prime}}\right)$. (If the underlying space of an almost complex manifold is a domain in a Euclidean space, we will call it the almost complex domain.) Assume that $\Omega$ is bounded and has smooth boundary. Regard $J$ and $J^{\prime}$ as matrixvalued functions on $\Omega$ and $\Omega^{\prime}$, respectively. In this section $j, k, l, \ldots=1,2, \ldots, 2 n$ and $\alpha, \beta, \gamma, \ldots=1,2, \ldots, 2 m$.

Let $f: \Omega \rightarrow \Omega^{\prime}$ be a pseudo-holomorphic mapping of class $C^{1}(\bar{\Omega})$. Then $J_{f}^{\prime}=$ $J^{\prime} \circ f$ is $2 m \times 2 m$ matrix-valued function defined on $\Omega$ of class $C^{1}(\bar{\Omega})$. We will fix $f$ and simply denote $J_{f}^{\prime}$ by $J^{\prime}$ for the rest of this section. Let $J=\left(a_{j}^{k}\right)$ and $J^{\prime}=\left(b_{\beta}^{\alpha}\right)$, where $a_{j}^{k} \in C^{\infty}(\bar{\Omega})$ and $b_{\beta}^{\alpha} \in C^{1}(\bar{\Omega})$.

Denote by $L^{2}\left(\Omega, \mathbb{R}^{2 m}\right)\left(\right.$ resp. $\left.L^{2}\left(\Omega, M_{2 m \times 2 n}(\mathbb{R})\right)\right)$ the space of $\mathbb{R}^{2 m}$-valued (resp. $2 m \times 2 n$ matrix-valued) square integrable functions. For $g \in L^{2}\left(\Omega, \mathbb{R}^{2 m}\right)$ and $\varphi \in L^{2}\left(\Omega, M_{2 m \times 2 n}(\mathbb{R})\right)$, we write $g=\left(g_{\alpha}\right)$ and $\varphi=\left(\varphi_{j}^{\alpha}\right)$. Define the inner products of $L^{2}\left(\Omega, \mathbb{R}^{2 m}\right)$ and $L^{2}\left(\Omega, M_{2 m \times 2 n}(\mathbb{R})\right)$ by

$$
\begin{aligned}
(g, h) & =\int_{\Omega}\left(\sum_{\alpha} g_{\alpha} h_{\alpha}\right), \\
(\varphi, \psi) & =\int_{\Omega} \operatorname{trace}\left(\varphi^{t} \psi+\left(J^{\prime} \varphi\right)^{t} J^{\prime} \psi\right) \\
& =\int_{\Omega}\left(\sum_{\alpha, j} \varphi_{j}^{\alpha} \psi_{j}^{\alpha}+\sum_{\alpha, \beta, \gamma, j} \varphi_{j}^{\alpha} b_{\alpha}^{\beta} b_{\gamma}^{\beta} \psi_{j}^{\gamma}\right),
\end{aligned}
$$

where $g, h \in L^{2}\left(\Omega, \mathbb{R}^{2 m}\right)$ and $\varphi, \psi \in L^{2}\left(\Omega, M_{2 m \times 2 n}(\mathbb{R})\right)$.

For fixed $f$, we can define the densely defined linear differential operator $\bar{\partial}$ : $L^{2}\left(\Omega, \mathbb{R}^{2 m}\right) \rightarrow L^{2}\left(\Omega, M_{2 m \times 2 n}(\mathbb{R})\right)$ by

$$
\bar{\partial} g=d g+J^{\prime} d g J
$$

where $d g$ denotes the Jacobian matrix of $g$. Since $f$ satisfies equation (1.1), it follows that $\bar{\partial} f=0$. The $(\alpha, j)$-th entry of $\bar{\partial} g$ can be expressed by

$$
(\bar{\partial} g)_{j}^{\alpha}=\frac{\partial g_{\alpha}}{\partial x_{j}}+\sum_{\beta, k} b_{\beta}^{\alpha} \frac{\partial g_{\beta}}{\partial x_{k}} a_{j}^{k}
$$

We consider the following linear differential operator $\vartheta: L^{2}\left(\Omega, M_{2 m \times 2 n}(\mathbb{R})\right) \rightarrow$ $L^{2}\left(\Omega, \mathbb{R}^{2 m}\right)$ by

$$
(\vartheta \varphi)_{\alpha}=-\sum_{j} \frac{\partial \varphi_{j}^{\alpha}}{\partial x_{j}}+\sum_{\beta, j, k} b_{\beta}^{\alpha} a_{j}^{k} \frac{\partial \varphi_{j}^{\beta}}{\partial x_{k}} .
$$

In fact, the principal part of the formal adjoint operator of $\bar{\partial}$ is of the form $\left(I+J^{\prime t} J^{\prime}\right) \vartheta$. Replacing $\varphi$ by $\bar{\partial} g$, we have

$$
(\vartheta \bar{\partial} g)_{\alpha}=-\sum_{j} \frac{\partial}{\partial x_{j}}(\bar{\partial} g)_{j}^{\alpha}+\sum_{\beta, j, k} b_{\beta}^{\alpha} a_{j}^{k} \frac{\partial}{\partial x_{k}}(\bar{\partial} g)_{j}^{\beta} .
$$


Applying equation (2.1), we have that

$$
\begin{aligned}
(\vartheta \bar{\partial} g)_{\alpha}= & -\sum_{j} \frac{\partial^{2} g_{\alpha}}{\partial x_{j} \partial x_{j}} \\
& -\sum_{\beta, j, k} b_{\beta}^{\alpha} a_{j}^{k}\left(\frac{\partial^{2} g_{\beta}}{\partial x_{j} \partial x_{k}}-\frac{\partial^{2} g_{\beta}}{\partial x_{k} \partial x_{j}}\right) \\
& +\sum_{\beta, \gamma, j, k, l} b_{\beta}^{\alpha} b_{\gamma}^{\beta} a_{j}^{k} a_{j}^{l} \frac{\partial^{2} g_{\gamma}}{\partial x_{k} \partial x_{l}} \\
& +(C g)_{\alpha},
\end{aligned}
$$

where $(C g)_{\alpha}$ is part of $(\vartheta \bar{\partial} g)_{\alpha}$ of lower order given by

$$
\begin{aligned}
(C g)_{\alpha}= & -\sum_{\beta, j, k} \frac{\partial g_{\beta}}{\partial x_{k}} \frac{\partial}{\partial x_{j}}\left(b_{\beta}^{\alpha} a_{j}^{k}\right) \\
& +\sum_{\beta, \gamma, j, k, l} b_{\beta}^{\alpha} a_{j}^{k} \frac{\partial g_{\gamma}}{\partial x_{l}} \frac{\partial}{\partial x_{k}}\left(b_{\gamma}^{\beta} a_{j}^{l}\right) .
\end{aligned}
$$

Remark 2.1. Since $a_{j}^{k}, b_{\beta}^{\alpha}$ and its first derivatives are continuous on $\bar{\Omega}$, it follows that $(C g)_{\alpha} \in L^{2}(\Omega)$ if $g \in W^{1,2}\left(\Omega, \mathbb{R}^{2 m}\right)=\bigoplus^{2 m} W^{1,2}(\Omega)$. In particular, $(C f)_{\alpha} \in L^{p}(\Omega)$ for any $p \geq 1$.

Let $p>2 n$. For any positive integer $k$, we have $k p>2 n$; hence by Theorem 5.23 in 1 ,,$W^{k, p}(\Omega)$ is a Banach algebra, i.e. $u v \in W^{k, p}(\Omega)$ for any $u$ and $v$ in $W^{k, p}(\Omega)$. Additionally, using the chain rule, $b_{\beta}^{\alpha} \in W^{k, p}(\Omega)$ whenever $f_{\alpha} \in W^{k, p}(\Omega)$ for each $\alpha$. Moreover, $(C f)_{\alpha} \in W^{k-1, p}(\Omega)$.

For convenience, we let $A_{l}^{k}=\sum_{j} a_{j}^{k} a_{j}^{l} \in C^{\infty}(\bar{\Omega})$. In fact, $A_{l}^{k}$ is the $(k, l)$-th entry of the matrix $J J^{t}$. Since $\sum_{\beta} b_{\beta}^{\alpha} b_{\gamma}^{\beta}=-\delta_{\alpha, \gamma}$, it follows that

$$
\begin{aligned}
(\vartheta \bar{\partial} g)_{\alpha}= & -\sum_{j} \frac{\partial}{\partial x_{j}}(\bar{\partial} g)_{j}^{\alpha}+\sum_{\beta, j, k} b_{\beta}^{\alpha} a_{j}^{k} \frac{\partial}{\partial x_{k}}(\bar{\partial} g)_{j}^{\beta} \\
= & -\sum_{j} \frac{\partial^{2} g_{\alpha}}{\partial x_{j} \partial x_{j}}-\sum_{k, l} A_{l}^{k} \frac{\partial^{2} g_{\alpha}}{\partial x_{k} \partial x_{l}} \\
& +(C g)_{\alpha}
\end{aligned}
$$

when each $g_{\alpha}$ is of class $C^{\infty}$. For any $h \in C_{0}^{1}(\Omega)$, we obtain

$$
\begin{aligned}
\int_{\Omega}(\vartheta \bar{\partial} g)_{\alpha} h= & \sum_{j} \int_{\Omega}(\bar{\partial} g)_{j}^{\alpha} \frac{\partial h}{\partial x_{j}}-\sum_{\beta, j, k} \int_{\Omega}(\bar{\partial} g)_{j}^{\beta} \frac{\partial}{\partial x_{k}}\left(b_{\beta}^{\alpha} a_{j}^{k} h\right) \\
= & \sum_{j} \int_{\Omega} \frac{\partial g_{\alpha}}{\partial x_{j}} \frac{\partial h}{\partial x_{j}}+\sum_{k, l} \int_{\Omega} \frac{\partial g_{\alpha}}{\partial x_{l}} \frac{\partial}{\partial x_{k}}\left(A_{l}^{k} h\right) \\
& +\int_{\Omega}(C g)_{\alpha} h .
\end{aligned}
$$

Since $C^{\infty}(\Omega)$ is dense in $W^{1,2}(\Omega)$, we take a sequence $f^{\nu}$ in $C^{\infty}\left(\Omega, \mathbb{R}^{2 m}\right)$ which converges to $f$ in $W^{1,2}\left(\Omega, \mathbb{R}^{2 m}\right)$. Then $\left(\bar{\partial} f^{\nu}\right)_{j}^{\alpha},\left(C f^{\nu}\right)_{\alpha}$ and all the remaining first derivatives of $f^{\nu}$ converge to those of $f$ in $L^{2}(\Omega)$. Since $(\bar{\partial} f)_{j}^{\alpha}=0$, the sequence of 
equations (2.2) for $f^{\nu}$ converges to

$$
-\sum_{j} \int_{\Omega} \frac{\partial f_{\alpha}}{\partial x_{j}} \frac{\partial h}{\partial x_{j}}-\sum_{k, l} \int_{\Omega} \frac{\partial f_{\alpha}}{\partial x_{l}} \frac{\partial}{\partial x_{k}}\left(A_{l}^{k} h\right)=\int_{\Omega}(C f)_{\alpha} h
$$

for any $h \in C_{0}^{1}(\Omega)$.

Take the linear partial differential operator $H=\sum_{j} \frac{\partial^{2}}{\partial x_{j} \partial x_{j}}+\sum_{k, l} A_{l}^{k} \frac{\partial^{2}}{\partial x_{k} \partial x_{l}}$. The symbol of $H$ is $\sum_{j} \zeta_{j}^{2}+\sum_{k, l} \zeta_{k} A_{l}^{k} \zeta_{l}=|\zeta|^{2}+|J \zeta|^{2}$. So $H$ is strictly elliptic on $\Omega$ with smooth coefficients. Equation (2.3) means that

$$
H f_{\alpha}=(C f)_{\alpha}
$$

in the weak sense.

By our assumption, it follows that $(C f)_{\alpha} \in L^{2}(\Omega)$ for each $\alpha$. By the elliptic regularity theorem (Theorem 8.8 in $\left[5\right.$ ), we have $f_{\alpha} \in W_{l o c}^{2,2}(\Omega)$ for each $\alpha$.

Let $p>2 n$. Since $(C f)_{\alpha} \in L^{p}(\Omega)$, by the uniqueness of solutions of the Dirichlet problem for the elliptic equation (Corollary 9.18 in [5]), it follows that $f_{\alpha} \in W_{l o c}^{2, p}(\Omega) \cap C^{0}(\bar{\Omega})$ for each $\alpha$. From Remark 2.1, we have $(C f)_{\alpha} \in W_{l o c}^{1, p}(\Omega)$; hence Theorem 9.19 in [5] implies that $f_{\alpha} \in W_{l o c}^{3, p}(\Omega)$ for each $\alpha$. Simultaneously, $(C f)_{\alpha} \in W_{l o c}^{2, p}(\Omega)$. Repeating our argument, we show that $f_{\alpha} \in W_{l o c}^{k, p}(\Omega)$ for each positive integer $k$. By the Sobolev imbedding theorem, we have

Proposition 2.2. Let $\left(M^{2 n}, J\right)$ and $\left(M^{\prime 2 m}, J^{\prime}\right)$ be $C^{\infty}$-smooth almost complex manifolds. Any $C^{1}$ pseudo-holomorphic mapping from $M$ to $M^{\prime}$ is of class $C^{\infty}$.

For the regularity of pseudo-holomorphic curves $(n=1)$, see Theorem 3.2.2 in [12] and Theorem 2.2.1 in [17.

\section{First order estimate of PSEUdo-holomorphic MAPPings}

In this section, we derive the Cauchy estimate for pseudo-holomorphic mappings. For the first order estimate, it suffices to treat the case of pseudo-holomorphic discs.

Proposition 3.1 (Sikorav [17]). Fix $r, \eta \in(0,1)$. Let $W$ be a bounded domain in $\mathbb{C}^{n}$. Then there exist positive constants $\varepsilon$ and $C$ with the following property:

If $\phi: \mathbf{D} \rightarrow W$ is a differentiable mapping such that

$$
\frac{\partial \phi}{\partial \bar{z}}+q(\phi) \frac{\partial \phi}{\partial z}=0
$$

where $q: W \rightarrow \operatorname{End}_{\mathbb{R}}\left(\mathbb{C}^{n}\right)$ is of class $C^{r}$ and $\|q\|_{C^{r}} \leq \varepsilon$, then $\phi$ is of class $C^{1+r}$ on $\mathbf{D}(1-\eta)$. Moreover,

$$
\|\phi\|_{C^{1+r}(\mathbf{D}(1-\eta))} \leq C\|\phi\|_{L^{\infty}} .
$$

The $C^{0}$ and $C^{k}$ norms for a $C^{k}$ mapping $f: U \subset \mathbb{R}^{n} \rightarrow \mathbb{R}^{m}$ is usually defined by $\|f\|_{C^{0}(U)}=\sum_{j=1}^{m} \sup _{x \in U}\left|f_{j}(x)\right|$ and $\|f\|_{C^{k}(U)}=\sum_{j=1}^{m} \sum_{|\alpha| \leq k}\left\|D^{\alpha} f_{j}\right\|_{C^{0}(U)}$, where $|\cdot|$ is a standard Euclidean norm. For $0<r<1$, the $C^{k+r}$ (Hölder) norm is defined by

$$
\|f\|_{C^{k+r}(U)}=\|f\|_{C^{k}(U)}+\sum_{j=1}^{m} \sup _{|\alpha|=k} \sup _{\substack{x \neq y \\ x, y \in U}} \frac{\left|D^{\alpha} f_{j}(x)-D^{\alpha} f_{j}(y)\right|}{|x-y|^{r}} .
$$


Note that for a $C^{1}$ mapping $f: U \subset \mathbb{R}^{n} \rightarrow \mathbb{R}^{m},\|f\|_{C^{1}(U)}$ is equivalent to

$$
\|f\|_{C^{0}(U)}+\sup _{\substack{v \in T U \\|v| \leq 1}}|d f(v)| .
$$

Now we present:

Theorem 3.2. Let $(\Omega, J) \subset \mathbb{R}^{2 n}$ and $\left(\Omega^{\prime}, J^{\prime}\right) \subset \mathbb{R}^{2 m}$ be almost complex domains. For each point $p \in \Omega^{\prime}$, there is a bounded neighborhood $U$ of $p$ in $\Omega^{\prime}$ such that $\left\{\|f\|_{C^{1}(K)}: f \in \mathcal{O}_{\left(J, J^{\prime}\right)}(\Omega, U)\right\}$ is uniformly bounded for any compact subset $K$ of $\Omega$.

Proof. First, let us study the pseudo-holomorphic discs in $\Omega^{\prime}$. Applying a linear change of coordinates and a translation of $\mathbb{R}^{2 m}$, we may assume that $p=0$ and $J^{\prime}$ coincides with the canonical complex structure at 0 , i.e. $J_{0}^{\prime}=J_{s t}$. Take a neighborhood $V$ of 0 such that $J^{\prime}+J_{s t}$ is invertible on $V$.

Suppose that $\phi: \mathbf{D} \rightarrow V \subset \Omega^{\prime}$ is a pseudo-holomorphic disc. Then the following equation holds:

$$
\frac{\partial \phi}{\partial y}=J_{\phi}^{\prime} \frac{\partial \phi}{\partial x}
$$

Since $\frac{\partial \phi}{\partial x}=\frac{\partial \phi}{\partial z}+\frac{\partial \phi}{\partial \bar{z}}$ and $\frac{\partial \phi}{\partial y}=J_{s t}\left(\frac{\partial \phi}{\partial z}-\frac{\partial \phi}{\partial \bar{z}}\right)$, we have

$$
\left(J_{\phi}^{\prime}+J_{s t}\right) \frac{\partial \phi}{\partial \bar{z}}=-\left(J_{\phi}^{\prime}-J_{s t}\right) \frac{\partial \phi}{\partial z} .
$$

Defining the mapping $q: V \rightarrow \operatorname{End}_{\mathbb{R}}\left(\mathbb{C}^{m}\right)$ by $q(a)=\left(J_{a}^{\prime}+J_{s t}\right)^{-1}\left(J_{a}^{\prime}-J_{s t}\right)$, we see that (3.1) can be written as

$$
\frac{\partial \phi}{\partial \bar{z}}+q(\phi) \frac{\partial \phi}{\partial z}=0
$$

Since $V$ is relatively compact in $\Omega^{\prime}, q$ has the same (Hölder) regularity as that of $J^{\prime}$ on $V$.

Define the renormalization $q_{\beta}$ of $q$ by $q_{\beta}: \beta^{-1} V=\left\{\beta^{-1} a: a \in V\right\} \rightarrow \operatorname{End}_{\mathbb{R}}\left(\mathbb{C}^{m}\right)$ and $q_{\beta}(a)=q(\beta a)$ for an arbitrary real number $\beta>0$. Take a sufficiently small $\beta$ such that $B(0,1) \subset \beta^{-1} V$, equivalently $B(0, \beta) \subset V$. Then for fixed $0<r<1$, we have

$$
\begin{aligned}
\left\|q_{\beta}\right\|_{C^{r}(B(0,1))} & =\left\|q_{\beta}\right\|_{C^{0}(B(0,1))}+\sup _{\substack{x \neq y \\
x, y \in B(0,1)}} \frac{\left|q_{\beta}(x)-q_{\beta}(y)\right|}{|x-y|^{r}} \\
& =\|q\|_{C^{0}(B(0, \beta))}+\sup _{\substack{x \neq y \\
x, y \in B(0,1)}} \frac{|q(\beta x)-q(\beta y)|}{|\beta x-\beta y|^{r}} \beta^{r} \\
& \leq\|q\|_{C^{0}(B(0, \beta))}+\sup _{\substack{x \neq y \\
x, y \in V}} \frac{|q(x)-q(y)|}{|x-y|^{r}} \beta^{r} .
\end{aligned}
$$

Since $q(0)=0$, it follows that $\|q\|_{C^{0}(B(0, \beta))} \rightarrow 0$ as $\beta \rightarrow 0$. For a sufficiently small $\beta$, we have that $\left\|q_{\beta}\right\|_{C^{r}(B(0,1))}<\varepsilon$, where $\varepsilon$ is in Proposition 3.1 for the case $W=B(0,1)$. Now a new mapping $\phi_{\beta}=\beta^{-1} \phi$ satisfies

$$
\frac{\partial \phi_{\beta}}{\partial \bar{z}}+q_{\beta}\left(\phi_{\beta}\right) \frac{\partial \phi_{\beta}}{\partial z}=0 .
$$


Let $U=B(0, \beta)$. By Proposition 3.1, we can deduce that

$$
\begin{aligned}
\|\phi\|_{C^{1}(\mathbf{D}(1-\eta))} & \leq \beta\left\|\phi_{\beta}\right\|_{C^{1+r}(\mathbf{D}(1-\eta))} \\
& \leq C \beta\left\|\phi_{\beta}\right\|_{L^{\infty}} \\
& \leq C\|\phi\|_{L^{\infty}}
\end{aligned}
$$

for any $\phi \in \mathcal{O}_{\left(J_{s t}, J^{\prime}\right)}(\mathbf{D}, U)$.

By 5.4a in [15], there is a constant $R>0$ such that for any vector $v \in T \Omega$ based on $K$ with $|v| \leq R$, there is a pseudo-holomorphic disc $\phi: \mathbf{D} \rightarrow \Omega$ such that $d \phi(\mathbf{e})=v$, where $\mathbf{e}$ is an unit vector in $T_{0} \mathbf{D}$. For any $f \in \mathcal{O}_{\left(J, J^{\prime}\right)}(\Omega, U)$, $f \circ \phi: \mathbf{D} \rightarrow U$ is pseudo-holomorphic; hence it follows that $|d f(v)|=|d(f \circ \phi)(\mathbf{e})| \leq$ $\left\|d(f \circ \phi)_{0}\right\| \leq\|f \circ \phi\|_{C^{1}(\mathbf{D}(1-\eta))} \leq C\|f \circ \phi\|_{L^{\infty}} \leq C\|f\|_{C^{0}}$. Therefore we have

$$
\begin{aligned}
\|f\|_{C^{1}(K)} & \sim\|f\|_{C^{0}(K)}+\sup _{x \in K} \sup _{\substack{v \in T_{x} \Omega \\
|v| \leq R}} \frac{1}{R}\left|d f_{x}(v)\right| \\
& \leq\|f\|_{C^{0}(\Omega)}+\frac{C}{R}\|f\|_{C^{0}(\Omega)} \\
& \leq\left(1+\frac{C}{R}\right)\|f\|_{C^{0}(\Omega)} .
\end{aligned}
$$

This proves the theorem.

\section{Pseudo-holomorphic Jet Bundles}

In order to prove Theorem 1.2, we need some information about the $\infty$-jet of a certain family of pseudo-holomorphic mappings at a given point. These can be obtained by jet bundles.

Gauduchon (4] ) has shown that there is a natural almost complex structure in a pseudo-holomorphic 1-jet bundle such that the lifting of the pseudo-holomorphic mapping is also pseudo-holomorphic. In the first two subsections, we follow Gauduchon's work (see chapter 4 in [2] and [4]).

4.1. Horizontal distribution. Let $\pi: E \rightarrow M$ be a vector bundle with a linear connection $\nabla$. For any point $u \in E_{x}=\pi^{-1}(x)$, the vertical tangent space $T_{u}^{V} E$ at $u$ is a subspace of $T_{u} E$ whose elements are tangent to $E_{x}$. Let $T^{V} E=\bigcup_{u \in E} T_{u}^{V} E$.

Fix any section $\xi \in \Gamma(E)$ with $\xi(x)=u$. For each vector $X \in T_{x} M$, we define a lifting $\widetilde{X}_{u}$ in $T_{u} E$ by

$$
\widetilde{X}_{u}=d \xi_{x}(X)-\nabla_{X} \xi
$$

where $\nabla_{X} \xi \in E_{x}$ is considered as an element of $T_{u}^{V} E$. This definition of $\widetilde{X}_{u}$ is independent of the choices for $\xi$. Therefore, the horizontal subspace $H_{u}^{\nabla}$ at $u$ can be uniquely defined as a lifting subspace of $T_{x} M$ in $T_{u} E$ up to the linear connection $\nabla$. We call $H^{\nabla}=\bigcup_{u \in E} H_{u}^{\nabla}$ the horizontal distribution. It is easy to check that $H^{\nabla}$ is a smooth distribution and that the following properties hold:

(a) $T_{u} E=H_{u}^{\nabla} \oplus T_{u}^{V} E$ at each $u \in E$.

(b) Let $v^{\nabla}: H^{\nabla} \oplus T^{V} E \rightarrow T^{V} E$ be a natural projection (vertical projection). If $Y \in T_{u} E$ with $d \xi_{x}(X)=Y$ for some section $\xi$, then

$$
v^{\nabla}(Y)=\nabla_{X} \xi
$$

(c) The vertical projection $v^{\nabla}$ is also smooth. This means that for any smooth vector field $X$ of $T E, v^{\nabla}(X)$ is a smooth vector field of $T^{V} E$. 
(d) Given $Y \in T_{u} E \backslash T_{u}^{V} E$, there is a unique vector $X \in T_{x} M$ such that $d \xi_{x}(X)=Y$ for some section $\xi$. Therefore we have the natural projection from $T_{u} E$ to $T_{x} M$ and the canonical decomposition $T_{u} E \simeq T_{\pi(u)} M \times T_{u}^{V} E$.

4.2. Pseudo-holomorphic 1-jet bundle and its almost complex structure. Given two smooth $\left(C^{\infty}\right)$ almost complex manifolds $\left(M^{2 n}, J\right)$ and $\left(M^{\prime 2 m}, J^{\prime}\right)$, a $\left(J, J^{\prime}\right.$ )-holomorphic (or pseudo-holomorphic) 1-jet bundle over $M \times M^{\prime}$ is defined by

$$
\mathcal{J}_{\left(J, J^{\prime}\right)}^{1}\left(M, M^{\prime}\right)=\bigcup_{(x, y) \in M \times M^{\prime}} \operatorname{Hom}_{\left(J_{x}, J_{y}^{\prime}\right)}\left(T_{x} M, T_{y} M^{\prime}\right),
$$

where $\operatorname{Hom}_{\left(J_{x}, J_{y}^{\prime}\right)}\left(T_{x} M, T_{y} M^{\prime}\right)$ is the space of $\left(J_{x}, J_{y}^{\prime}\right)$-linear transformations from $T_{x} M$ to $T_{y} M^{\prime}$. Now $\pi=\pi_{1} \times \pi_{2}: \mathcal{J}_{\left(J, J^{\prime}\right)}^{1}\left(M, M^{\prime}\right) \rightarrow M \times M^{\prime}$ is a vector bundle of rank $2 \mathrm{~nm}$. We will frequently use the notation $\mathcal{J}^{1}\left(M, M^{\prime}\right)$ instead of $\mathcal{J}_{\left(J, J^{\prime}\right)}^{1}\left(M, M^{\prime}\right)$ for simplicity.

Choose any linear connection $\nabla$ on $\mathcal{J}^{1}\left(M, M^{\prime}\right)$. We have the canonical identification

$$
\begin{aligned}
T_{u} \mathcal{J}^{1}\left(M, M^{\prime}\right) & \simeq T_{\pi_{1}(u)} M \times T_{\pi_{2}(u)} M^{\prime} \times T_{u}^{V} \mathcal{J}^{1}\left(M, M^{\prime}\right) \\
& \simeq T_{\pi_{1}(u)} M \times T_{\pi_{2}(u)} M^{\prime} \times \operatorname{Hom}_{\left(J, J^{\prime}\right)}\left(T_{\pi_{1}(u)} M, T_{\pi_{2}(u)} M^{\prime}\right) .
\end{aligned}
$$

By this, any tangent vector $Y \in T_{u} \mathcal{J}^{1}\left(M, M^{\prime}\right)$ can be decomposed into

$$
Y=\left(X_{1}, X_{2}, v^{\nabla}(Y)\right)
$$

where:

i) $X_{1}$ and $X_{2}$ are images of the natural projection of $Y$ into $T_{\pi_{1}(u)} M$ and $T_{\pi_{2}(u)} M^{\prime}$, respectively,

ii) $v^{\nabla}(Y)$ is considered as an element in $\operatorname{Hom}_{\left(J, J^{\prime}\right)}\left(T_{\pi_{1}(u)} M, T_{\pi_{2}(u)} M^{\prime}\right)$.

Now we can define an almost complex structure $J^{\nabla}$ on $\mathcal{J}^{1}\left(M, M^{\prime}\right)$ depending on $\nabla$ by

$$
J^{\nabla}(Y)=\left(J_{\pi_{1}(u)} X_{1}, J_{\pi_{2}(u)}^{\prime} X_{2}, J_{\pi_{2}(u)}^{\prime} \circ v^{\nabla}(Y)\right) .
$$

It is easy to see $v^{\nabla}\left(J^{\nabla}(Y)\right)=J_{\pi_{2}(u)}^{\prime} \circ v^{\nabla}(Y)$; hence $J^{\nabla}$ is well defined. Furthermore, $J^{\nabla}$ is a smooth almost complex structure. Hence $\left(\mathcal{J}^{1}\left(M, M^{\prime}\right), J^{\nabla}\right)$ is also a smooth almost complex manifold.

Theorem 4.1 (Gauduchon [4]). There is a linear connection $\nabla$ on $\mathcal{J}^{1}\left(M, M^{\prime}\right)$ with following property:

For any pseudo-holomorphic mapping $f: M \rightarrow M^{\prime}$, its lifting $\mathrm{L}(f):(M, J) \rightarrow$ $\left(\mathcal{J}^{1}\left(M, M^{\prime}\right), J^{\nabla}\right)$ is also pseudo-holomorphic.

4.3. Higher order jet bundles. We can define the $k$-jet bundles over $M \times M^{\prime}$ inductively. But we need only the local information, so we shall consider the Euclidean case.

Let $(\Omega, J) \subset \mathbb{R}^{2 n}$ and $\left(\Omega^{\prime}, J^{\prime}\right) \subset \mathbb{R}^{2 m}$ be smooth almost complex domains. Let $\left(x_{1}, \ldots, x_{2 n}\right)$ and $\left(w_{1}, \ldots, w_{2 m}\right)$ be the standard coordinate systems for $\mathbb{R}^{2 n}$ and $\mathbb{R}^{2 m}$, respectively. Assume that

(*) $\quad\left\{\partial / \partial x_{1}, \cdots, \partial / \partial x_{n}\right\}$ is a complex basis of $T_{x} \Omega$ for each $x \in \Omega$.

Condition $(*)$ means that $\left\{\partial / \partial x_{1}, \ldots, \partial / \partial x_{n}\right\}$ and its images under $J_{x}$ form a real basis of $T_{x} \Omega$. 
By $(*)$ a $\left(J, J^{\prime}\right)$-linear mapping from $T_{x} \Omega$ to $T_{y} \Omega^{\prime}$ is completely determined by the images of $\left\{\partial / \partial x_{1}, \ldots, \partial / \partial x_{n}\right\}$; hence $\mathcal{J}^{1}\left(\Omega, \Omega^{\prime}\right)$ is a trivial bundle. From now on, we consider $\mathcal{J}^{1}\left(\Omega, \Omega^{\prime}\right)$ as an open set $\Omega \times \Omega^{\prime} \times \mathbb{R}^{2 n m}$ in $\mathbb{R}^{2(n+m+n m)}$. More precisely, a coordinate mapping is given by

$$
\tau=\left(\pi_{1}(\tau), \pi_{2}(\tau),\left[d w_{\alpha}\left(\tau\left(\frac{\partial}{\partial x_{j}}\right)\right)\right]_{\substack{\alpha=1, \ldots, 2 m \\ j=1, \ldots, n}}\right)
$$

The lifting $\mathrm{L}(f)$ of a pseudo-holomorphic mapping $f$ is parameterized by

$$
\mathrm{L}(f)(x)=\left(x_{1}, \ldots, x_{2 n}, f_{1}(x), \ldots, f_{2 m}(x),\left[\frac{\partial f_{\alpha}}{\partial x_{j}}(x)\right]_{\substack{\alpha=1, \ldots, 2 m \\ j=1, \ldots, n}}\right) .
$$

To compare $\|f\|_{C^{l}}$ with $\|\mathrm{L}(f)\|_{C^{l-1}}$, we have to consider the partial derivatives of $f$ that are missing in the above expression of $\mathrm{L}(f)(x)$. Solving the system of linear equations $J_{f}^{\prime} \circ d f=d f \circ J$ with respect to $\left\{\partial f_{\alpha} / \partial x_{j}\right\}_{j>n}$, we have

$$
\frac{\partial f_{\alpha}}{\partial x_{j}}(x)=\sum_{\beta=1}^{2 m} \sum_{k=1}^{n} A_{j k}^{\alpha \beta}(x, f(x)) \frac{\partial f_{\beta}}{\partial x_{k}}(x) \quad \text { on } \Omega
$$

for $j>n$, where $A_{j k}^{\alpha \beta}$ is a globally defined $C^{\infty}$-smooth function on $\Omega \times \Omega^{\prime}$. Therefore, for each compact subset $K$ in $\Omega$ and any positive integer $l$, there is a suitable constant $M_{l}$ depending on $K$ with

$$
\left\|\frac{\partial f_{\alpha}}{\partial x_{j}}\right\|_{C^{l}(K)} \leq M_{l} \sum_{\beta=1}^{2 m} \sum_{k=1}^{n}\left\|\frac{\partial f_{\beta}}{\partial x_{k}}\right\|_{C^{l}(K)}
$$

for $j>n$. We may deduce that

$$
\|f\|_{C^{l}(K)} \lesssim\|\mathrm{L}(f)\|_{C^{l-1}(K)}
$$

uniformly for $f \in \mathcal{O}_{\left(J, J^{\prime}\right)}\left(\Omega, \Omega^{\prime}\right)$.

By the expression (4.3), we also obtain

Proposition 4.2. Let $f, g \in \mathcal{O}_{\left(J, J^{\prime}\right)}\left(\Omega, \Omega^{\prime}\right)$ and $\nu \geq 1$. If $f$ and $g$ share the same $\nu$-jet at $p \in \Omega$, then $\mathrm{L}(f)$ and $\mathrm{L}(g)$ share the same $(\nu-1)$-jet at $p$.

We now go to the 2 -jet.

Take any linear connection $\nabla_{1}$ on $\mathcal{J}^{1}\left(\Omega, \Omega^{\prime}\right)$. From our assumption $(*)$ about $\Omega$, the pseudo-holomorphic 2 -jet bundle over $\Omega \times \Omega^{\prime}$ defined by

$$
\mathcal{J}^{2}\left(\Omega, \Omega^{\prime}\right)=\mathcal{J}_{\left(J, J^{\left.\nabla_{1}\right)}\right.}^{1}\left(\Omega, \mathcal{J}^{1}\left(\Omega, \Omega^{\prime}\right)\right)
$$

is also trivial. Choosing $\nabla_{\nu}$ inductively, we can define a pseudo-holomorphic $(\nu+1)$ jet bundle by

$$
\mathcal{J}^{\nu+1}\left(\Omega, \Omega^{\prime}\right)=\mathcal{J}_{\left(J, J^{\nabla_{\nu}}\right)}^{1}\left(\Omega, \mathcal{J}^{\nu}\left(\Omega, \Omega^{\prime}\right)\right)
$$

For any choice of $\nabla_{\nu}$ at each step, $\mathcal{J}^{\nu}\left(\Omega, \Omega^{\prime}\right)$ is always trivial.

From now on, we fix a suitable linear connection $\nabla_{\nu}$ as in Theorem 4.1 at each step. Then for a pseudo-holomorphic mapping $f: \Omega \rightarrow \Omega^{\prime}$, its lifting $\mathrm{L}^{\nu}(f)=$ $\mathrm{L}\left(\mathrm{L}^{\nu-1}(f)\right): \Omega \rightarrow \mathcal{J}^{\nu}\left(\Omega, \Omega^{\prime}\right)$ is always $\left(J, J^{\nabla_{\nu}}\right)$-holomorphic.

Given $f \in \mathcal{O}_{\left(J, J^{\prime}\right)}\left(\Omega, \Omega^{\prime}\right)$ and $p \in \Omega$, a family of mappings defined by

$$
\mathcal{F}_{p}^{\nu}\left(f ; \Omega, \Omega^{\prime}\right)=\left\{g \in \mathcal{O}_{\left(J, J^{\prime}\right)}\left(\Omega, \Omega^{\prime}\right): g \text { has the same } \nu \text {-jet with } f \text { at } p\right\}
$$

has the following property. 
Theorem 4.3. Let $(\Omega, J) \subset \mathbb{R}^{2 n}$ and $\left(\Omega^{\prime}, J^{\prime}\right) \subset \mathbb{R}^{2 m}$ be hyperbolic almost complex domains. Assume that $\Omega$ satisfies condition $(*)$. For any $f \in \mathcal{O}_{\left(J, J^{\prime}\right)}\left(\Omega, \Omega^{\prime}\right)$, there is a neighborhood $V_{\nu}$ of $p$ such that $\left\{\mathrm{L}^{\nu}(g): g \in \mathcal{F}_{p}^{\nu-1}\left(f ; \Omega, \Omega^{\prime}\right)\right\}$ is uniformly bounded on $V_{\nu}$. Moreover, we can find $V_{\nu}$ such that $V_{\nu+1} \subset \subset V_{\nu}$ for each $\nu=1,2, \ldots$

Proof. Choose $r>0$ such that the Kobayashi ball $U=\mathbf{B}_{\left(\Omega^{\prime}, J^{\prime}\right)}(f(p), r)$ is a bounded neighborhood of $f(p)$ as in Theorem 3.2. Denote $V=\mathbf{B}_{(\Omega, J)}(p, r)$. Since $\mathcal{F}_{p}^{0}\left(f ; \Omega, \Omega^{\prime}\right)=\left\{g \in \mathcal{O}_{\left(J, J^{\prime}\right)}\left(\Omega, \Omega^{\prime}\right): g(p)=f(p)\right\}$, we have $g(V) \subset U$ for any $g \in \mathcal{F}_{p}^{0}\left(f ; \Omega, \Omega^{\prime}\right)$. Take any relatively compact neighborhood $V_{1}$ of $p$ in $V$. By Theorem 3.2. $\left\{\|g\|_{C^{1}\left(V_{1}\right)}: g \in \mathcal{F}_{p}^{0}\left(f ; \Omega, \Omega^{\prime}\right)\right\}$ is uniformly bounded so that $\left\{\mathrm{L}(g): g \in \mathcal{F}_{p}^{0}\left(f ; \Omega, \Omega^{\prime}\right)\right\}$ is uniformly bounded on $V_{1}$. This proves the case $\nu=1$.

Since $(V, J)$ and $\left(U, J^{\prime}\right)$ are also Kobayashi hyperbolic, Theorem 3 in [1] implies that every bounded domain in $\mathcal{J}_{\left(J, J^{\prime}\right)}^{1}(V, U)$ is hyperbolic with respect to $J^{\nabla_{1}}$. Therefore, we may assume that

$$
\bigcup_{g \in \mathcal{F}_{p}^{0}\left(f ; \Omega, \Omega^{\prime}\right)} \mathrm{L}(g)\left(V_{1}\right) \subset \Omega_{1},
$$

where $\Omega_{1}$ is a hyperbolic neighborhood of $\mathrm{L}(f)(p)$ in $\mathcal{J}_{\left(J, J^{\prime}\right)}^{1}(V, U)$.

Suppose that our theorem holds for the case $\nu \leq \lambda$. Since the pair $\left(V_{1}, J\right)$ and $\left(\Omega_{1}, J^{\nabla_{1}}\right)$ satisfy the assumption of the theorem, there are neighborhoods $V_{1}^{\prime}, \ldots, V_{\lambda}^{\prime}$ of $p$ in $V_{1}$ such that $\left\{\mathrm{L}^{\nu}(h): h \in \mathcal{F}_{p}^{\nu-1}\left(\mathrm{~L}(f) ; V_{1}, \Omega_{1}\right)\right\}$ is uniformly bounded on $V_{\nu}^{\prime}$ for $\nu=1, \ldots, \lambda$, and such that $V_{\lambda}^{\prime} \subset \subset V_{\lambda-1}^{\prime} \subset \subset \cdots \subset \subset V_{1}^{\prime}$. By Proposition 4.2, we have

$$
\mathrm{L}\left(\mathcal{F}_{p}^{\nu}\left(f ; \Omega, \Omega^{\prime}\right)\right) \subset \mathcal{F}_{p}^{\nu-1}\left(\mathrm{~L}(f) ; V_{1}, \Omega_{1}\right)
$$

for any $\nu$. Therefore $\mathrm{L}^{\nu+1}(g)=\mathrm{L}^{\nu}(\mathrm{L}(g))$ is uniformly bounded on $V_{\nu+1}=V_{\nu}^{\prime}$ for $g \in \mathcal{F}_{p}^{\nu}\left(f ; \Omega, \Omega^{\prime}\right)$ and for $\nu=1, \ldots, \lambda$. This proves the theorem by the induction hypothesis.

For this sequence $\left\{V_{\nu}\right\}$ of nested neighborhoods of $p$, we have

Corollary 4.4. $\left\{\|g\|_{C^{\nu}\left(V_{\nu}\right)}: g \in \mathcal{F}_{p}^{\nu-1}\left(f ; \Omega, \Omega^{\prime}\right)\right\}$ is uniformly bounded.

Proof. From (4.4), we have

$$
\|g\|_{C^{\nu}\left(V_{\nu}\right)} \lesssim\|\mathrm{L}(g)\|_{C^{\nu-1}\left(V_{\nu}\right)} \lesssim \cdots \lesssim\left\|\mathrm{L}^{\nu}(g)\right\|_{C^{0}\left(V_{\nu}\right)}
$$

uniformly for $g \in \mathcal{O}_{\left(J, J^{\prime}\right)}\left(\Omega, \Omega^{\prime}\right)$. When $g \in \mathcal{F}_{p}^{\nu-1}\left(f ; \Omega, \Omega^{\prime}\right)$, the last term of this inequality is bounded by Theorem 4.3

\section{Proof of Theorem 1.2}

Let $(M, J)$ be a connected hyperbolic almost complex manifold of class $C^{\infty}$. Suppose that there is a pseudo-holomorphic self-mapping $f: M \rightarrow M$ with $f(p)=p$ and $d f_{p}=\operatorname{Id}$ for some $p \in M$. From Proposition [2.2, $f$ is of class $C^{\infty}$ and we can compare all partial derivatives of $f$ with those of the identity mapping. To prove that $f$ is the identity, we need the unique continuation property for pseudoholomorphic mappings.

Proposition 5.1. Let $(M, J)$ and $\left(M^{\prime}, J^{\prime}\right)$ be smooth almost complex manifolds. Moreover $M$ is connected. Suppose that two pseudo-holomorphic mappings $f, g$ : $M \rightarrow M^{\prime}$ share the same $\infty$-jet at some point in $M$. Then $f \equiv g$ on $M$. 
Proof. It is sufficient to prove that $A=\{p \in M: f$ and $g$ share the same $\infty$-jet at $p\}$ is open. Then our assertion follows, since $A$ is open, closed and nonempty set.

Suppose that $p \in A$. There is a neighborhood $U_{p}$ of $p$ such that any point $q$ in $U_{p}$ can be joined to $p$ by a single pseudo-holomorphic disc ([6] and [10]). Take any $q$ in $U_{p}$ and suppose that there is a pseudo-holomorphic disc $\phi: \mathbf{D} \rightarrow M$ with $\phi(0)=p$ and $\phi(1 / 2)=q$. Since $p \in A$, the two pseudo-holomorphic discs $f \circ \phi, g \circ \phi: \mathbf{D} \rightarrow M^{\prime}$ share the same $\infty$-jet at 0 . By the unique continuation property of pseudo-holomorphic curves (see [3] and [12]), it holds that $f \circ \phi \equiv g \circ \phi$. Furthermore $f(q)=g(q)$. Since $q$ is an arbitrary point in $U_{p}$, we have $\left.\left.f\right|_{U_{p}} \equiv g\right|_{U_{p}}$. Hence $p \in U_{p} \subset A$, and $A$ is open. This proves the proposition.

By Proposition [5.1, it is sufficient to prove that $D^{\alpha} f_{j}(p)=0$ for any $j$ and any multi-indices $|\alpha| \geq 2$. Then $f$ has the same $\infty$-jet with the identity mapping. Therefore $f$ is the identity mapping.

Choose a local coordinate system $\varphi:(V, 0) \rightarrow(M, p)$ about $p$ with $\varphi(V) \subset \subset M$. Since the Kobayashi distance function $d_{(M, J)}$ is continuous, we can take a positive real number $r<\min _{q \in \partial \varphi(V)} d_{(M, J)}(p, q)$. Then the Kobayashi ball $\mathbf{B}_{(M, J)}(p, r)$ is contained in $\varphi(V)$. By the distance-decreasing property of the Kobayashi distance, we have $f\left(\mathbf{B}_{(M, J)}(p, r)\right) \subset \mathbf{B}_{(M, J)}(p, r)$ for all $r$. Now we identify $p=0, \varphi(V)=$ $V$ is a bounded domain in $\mathbb{R}^{2 n}$ and $J=\varphi^{*} J=(d \varphi)^{-1} \circ J \circ d \varphi$ is an induced almost complex structure on $V$. For sufficiently small $r$ we may assume that ( $U=$ $\left.\varphi^{-1}\left(\mathbf{B}_{(M, J)}(p, r)\right), J\right)$ satisfies condition $(*)$ in Section 4 .

Consider an iterated family $\left\{f^{m}=f \circ f^{m-1}\right\}_{m=1,2, \ldots}$ of $f$. Note that $\left.f\right|_{U}$ is in $\mathcal{O}_{(J, J)}(U, U)$, so is $\left.f^{m}\right|_{U}$. Now we have

Proposition 5.2. $\left(D^{\alpha}\left(f^{m}\right)_{j}\right)(0)=m\left(D^{\alpha} f_{j}\right)(0)$ for $|\alpha|=2$.

Suppose that $D^{\alpha} f_{j}(0)=0$ for any $2 \leq|\alpha|<\nu$ and $j=1, \ldots, 2 n$. Then $\left(D^{\beta}\left(f^{m}\right)_{j}\right)(0)=m\left(D^{\beta} f_{j}\right)(0)$ for each $|\beta|=\nu$ and each $j$.

Proof. Since $d\left(f^{m}\right)_{0}=\left(d f_{0}\right)^{m}=\mathrm{Id}$, we have

$$
\frac{\partial\left(f^{m}\right)_{j}}{\partial x_{k}}(0)=\delta_{j, k}
$$

for $m=1,2, \ldots$.

Let $D^{\alpha}=\frac{\partial^{2}}{\partial x_{\alpha_{1}} \partial x_{\alpha_{2}}}$. Since $\left(f^{m}\right)_{j}=f_{j} \circ f^{m-1}$, we have

$$
\begin{aligned}
\frac{\partial^{2}}{\partial x_{\alpha_{1}} \partial x_{\alpha_{2}}}\left(f^{m}\right)_{j}(0)= & \frac{\partial}{\partial x_{\alpha_{1}}}\left(\sum_{k=1}^{2 n} \frac{\partial f_{j}}{\partial x_{k}}\left(f^{m-1}(x)\right) \frac{\partial\left(f^{m-1}\right)_{k}}{\partial x_{\alpha_{2}}}(x)\right)(0) \\
= & \sum_{k, l=1}^{2 n} \frac{\partial^{2} f_{j}}{\partial x_{l} \partial x_{k}}\left(f^{m-1}(0)\right) \frac{\partial\left(f^{m-1}\right)_{l}}{\partial x_{\alpha_{1}}}(0) \frac{\partial\left(f^{m-1}\right)_{k}}{\partial x_{\alpha_{2}}}(0) \\
& +\sum_{k=1}^{2 n} \frac{\partial f_{j}}{\partial x_{k}}\left(f^{m-1}(0)\right) \frac{\partial^{2}\left(f^{m-1}\right)_{k}}{\partial x_{\alpha_{1}} \partial x_{\alpha_{2}}}(0) \\
= & \frac{\partial^{2} f_{j}}{\partial x_{\alpha_{1}} \partial x_{\alpha_{2}}}(0)+\frac{\partial^{2}\left(f^{m-1}\right)_{j}}{\partial x_{\alpha_{1}} \partial x_{\alpha_{2}}}(0)
\end{aligned}
$$

where the last equality follows by (5.1). This equation proves the case of $|\alpha|=2$ by induction. 
Suppose that $D^{\alpha} f_{j}(0)=0$ for any $2 \leq|\alpha|<\nu$ and $j=1, \ldots, 2 n$. Let $|\beta|=\nu$ and $D^{\beta}=\frac{\partial^{\nu}}{\partial x_{\beta_{1}} \cdots \partial x_{\beta_{\nu}}}$. From (5.1), we obtain

$$
\begin{aligned}
D^{\beta}\left(f^{m}\right)_{j}(0) & \\
= & \sum_{\gamma_{1}, \ldots, \gamma_{\nu}=1}^{2 n} \frac{\partial^{\nu} f_{j}}{\partial x_{\gamma_{1}} \cdots \partial x_{\gamma_{\nu}}}\left(f^{m-1}(0)\right) \frac{\partial\left(f^{m-1}\right)_{\gamma_{1}}}{\partial x_{\beta_{1}}}(0) \cdots \frac{\partial\left(f^{m-1}\right)_{\gamma_{\nu}}}{\partial x_{\beta_{\nu}}}(0) \\
& +\left(\text { terms which contain } D^{\alpha} f_{j} \text { for } 2 \leq|\alpha|<\nu\right) \\
& +\sum_{k=1}^{2 n} \frac{\partial f_{j}}{\partial x_{k}}\left(f^{m-1}(0)\right) \frac{\partial^{\nu}\left(f^{m-1}\right)_{k}}{\partial x_{\beta_{1}} \cdots \partial x_{\beta_{\nu}}}(0) \\
= & \frac{\partial^{\nu} f_{j}}{\partial x_{\beta_{1}} \cdots \partial x_{\beta_{\nu}}}(0)+\frac{\partial^{\nu}\left(f^{m-1}\right)_{j}}{\partial x_{\beta_{1}} \cdots \partial x_{\beta_{\nu}}}(0) \\
= & D^{\beta} f_{j}(0)+D^{\beta}\left(f^{m-1}\right)_{j}(0) .
\end{aligned}
$$

This proves the proposition.

We are now ready to complete the proof of Theorem 1.2 .

Suppose that $D^{\alpha} f_{j}(0) \neq 0$ for some multi-index $\alpha$ with $|\alpha|=2$ and some $j$. By Proposition [5.2] we have $\left|\left(D^{\alpha}\left(f^{m}\right)_{j}\right)(0)\right|=m\left|\left(D^{\alpha} f_{j}\right)(0)\right| \rightarrow \infty$ as $m \rightarrow \infty$. Since $f^{m}(0)=f(0)=0$ and $d\left(f^{m}\right)_{0}=d f_{0}=\mathrm{Id}$, we have $f^{m} \in \mathcal{F}_{0}^{1}(f ; U, U)$ for each $m$. Corollary 4.4 implies that $\left\{\left|\left(D^{\alpha}\left(f^{m}\right)_{j}\right)(0)\right|\right\}_{m=1,2, \ldots}$ must be bounded. Therefore it follows that $D^{\alpha} f_{j}(0)=0$ for each $|\alpha|=2$ and $j$.

Inductively let us assume that $D^{\beta} f_{j}(0) \neq 0$ and $D^{\alpha} f_{k}(0)=0$ for $2 \leq|\alpha|<|\beta|=$ $\nu$ and $k=1, \ldots, 2 n$. Proposition 5.2 implies that $\left(D^{\alpha}\left(f^{m}\right)_{k}\right)(0)=m\left(\bar{D}^{\alpha} f_{k}\right)(0)=0$ for $2 \leq|\alpha|<\nu$ and $k=1, \ldots, 2 n$. Hence it follows that $f^{m} \in \mathcal{F}_{0}^{\nu-1}(f ; U, U)$. But Proposition 5.2 also means that $\left|\left(D^{\beta}\left(f^{m}\right)_{j}\right)(0)\right|=m\left|\left(D^{\beta} f_{j}\right)(0)\right| \rightarrow \infty$ as $m \rightarrow \infty$. It is a contradiction to Corollary 4.4. Therefore we have $D^{\alpha} f_{j}(0)=0$ for any $|\alpha| \geq 2$.

Consequently $f$ has same $\infty$-jet with the identity mapping at 0 . This proves Theorem 1.2 .

\section{ACKNOWLEDGMENT}

This work is part of the author's dissertation for his doctoral degree at the Pohang University of Science and Technology. He expresses his gratitude to his advisor Kang-Tae Kim for guidance and encouragement.

\section{REFERENCES}

[1] R.A. Adams, Sobolev spaces, Pure and Applied Mathematics, Vol. 65, Academic Press, 1975. MR0450957 (56:9247)

[2] M. Audin, J. Lafontaine, editors, Holomorphic curves in symplectic geometry, Progress in Mathematics, 117, Birkhäuser Verlag, Basel, 1994. MR1274923 (95i:58005)

[3] A. Floer, H. Hofer, D. Salamon, Transversality in elliptic Morse theory for the symplectic action, Duke. Math. J., 80 (1995), 251-292. MR1360618 (96h:58024)

[4] P. Gauduchon, The canonical almost complex structure on the manifold of 1-jets of pseudoholomorphic mappings between two almost complex manifolds, appendix to Chap. II in [2]. MR:1274923 (95i:58005)

[5] D. Gilbarg, N.S. Trudinger, Elliptic partial differential equations of second order, Second edition. Grundlehren der Mathematischen Wissenschaften, 224, Springer-Verlag, Berlin, 1983. MR0737190 (86c:35035) 
[6] J. Globevnik, Perturbing analytic discs attached to maximal real submanifold of $\mathbb{C}^{N}$, Indag. Math., 7 (1996), 37-46. MR 1621348 (99b:32023)

[7] M. Gromov, Pseudo holomorphic curves in symplectic manifolds, Invent. Math., 82 (1985), $307-347$.

[8] S. Kobayashi, Hyperbolic manifolds and holomorphic mappings, Pure and Applied Mathematics, 2 Marcel Dekker, Inc., New York, 1970. MR0277770 (43:3503)

[9] S. Kobayashi, Hyperbolic complex spaces, Grundlehren der Mathematischen Wissenschaften, 318, Springer-Verlag, Berlin, 1998. MR1635983(99m:32026)

[10] B.S. Kruglikov, Existence of close pseudoholomorphic disks for almost complex manifolds and their application to the Kobayashi-Royden pseudonorm (Russian) Funktsional. Anal. i Prilozhen., 33 (1999), no. 1, 46-58, 96; translation in Funct. Anal. Appl., 33 (1999), no. 1, 38-48. MR 1711878 (2000i:32044)

[11] B.S. Kruglikov, M. Overholt, Pseudoholomorphic mappings and Kobayashi hyperbolicity, Differential Geom. Appl. 11 (1999), no. 3, 265-277. MR1726542 (2000j:32042)

[12] D. McDuff, D. Salamon, J-holomorphic curves and quantum cohomology, University Lecture Series, 6, American Mathematical Society, Providence, RI, 1994. MR1286255 (95g:58026)

[13] R. Narasimhan, Several complex variables, Chicago Lectures in Mathematics, The University of Chicago Press, Chicago, 1971. MR0342725 (49:7470)

[14] A. Newlander, L. Nirenberg, Complex analytic coordinates in almost complex manifolds, Ann. of Math., 65 (1957), 391-404. MR0088770 (19:577a)

[15] A. Nijenhuis, W. Woolf, Some integration problems in almost-complex and complex manifolds, Ann. Math., 77 (1963), 424-489. MR0149505(26:6992)

[16] H.L. Royden, Remarks on the Kobayashi metric, Several complex variables, II (Proc. Internat. Conf., Univ. Maryland, College Park, Md., 1970), pp. 125-137. Lecture Notes in Math., Vol. 185, Springer, Berlin, 1971. MR0304694 (46:3826)

[17] J.C. Sikorav, Some properties of holomorphic curves in almost complex manifolds, 165-189, in [2]. MR:1274929

Department of Mathematics, Pohang University of Science and Technology, Pohang, 790-784, Republic of Korea

E-mail address: nyawoo@postech.ac.kr

Current address: Department of Mathematical Sciences, Seoul National University, Seoul, 151-747, Republic of Korea 